\title{
PREDICTIVE INDICATORS FOR MANAGING DENTAL CARE IN PATIENTS WITH DIFFERENT ABILITIES
}

\author{
ABSTRACT \\ Objectives: To determine predictive indicators for dental health \\ management of patients with different abilities, according to a new \\ screening protocol.
}

Materials and methods: This was an observational, analytical, retrospective, cross-sectional study. The sample consisted of all patients with different abilities, diagnosed with autism, Down syndrome, cognitive deficit, cerebral palsy and others, who received care between 1999 and 2019 at the Oral Health Service of the Centro Ann Sullivan del Peru and met the inclusion criteria. Data were obtained from the clinical histories to determine the predictive indicators for managing dental care in these patients. A multivariate statistical analysis was performed using binary logistic regression test. The research project was evaluated by the Research Project Reviewing Committee and then referred to the Human Ethics Committee from a university, securing the relevant approvals.

Results: Of the 589 cases evaluated, mean age was 14.5 years, $67.9 \%$ were male; systemic diagnosis of different abilities was autism in $62 \%$, the most frequent treatment was caries $(52.1 \%)$; and final management of these patients was conscious in $96.4 \%$ of the cases. With regard to type of classification and horizon, $67.1 \%$ of the cases corresponded to classification A, with $35.9 \%$ of these in horizon I. With regard to diagnosis of different abilities, autism presented $40.2 \%$ of the cases in classification A and horizon I. A predictive equation of $97.1 \%$ probability of whether or not sedation was used was obtained. Regression analysis showed that this model has high sensitivity (100\%) and low specificity (15\%).

Conclusions: Findings show that the type of management for dental care was conscious in nearly all the cases dealt with. This was significantly influenced by the Classification and Horizon Screening Protocol indicator, which established the best predictive model for whether or not to use sedation. The model adequately classifies patients who do not require sedation.

Keywords: Dental care for disabled, dental anxiety, disease management, conscious sedation.

\author{
*Oswaldo Carlos José Cava- \\ Arangoitia ${ }^{1}$ \\ (D) Janet Ofelia Guevara-Canales ${ }^{2}$ \\ (D) Rafael Morales-Vadillo ${ }^{3}$ \\ (D) Carlos Enrique Cava-Vergiú ${ }^{4}$ \\ (D) Manuel Ribera Uribe ${ }^{5}$ \\ (D) Liliana Mayo Ortega ${ }^{6}$
}

ORCID IDs of the authors:

O.C.J.C.A. $\quad 0000-0001-8038-120 \mathrm{X}$

J.O.G.C. $\quad 0000-0002-7040-8269$

R.M.V. $\quad 0000-0002-7835-6408$

C.E.C.V.. $\quad 0000-0003-0338-3425$

M.R.U. 0000-0002-9919-3281

L.M.O. 0000-0002-9280-6137

${ }^{1}$ Dental Surgeon, Lecturer at the Pediatric Dentistry Postgraduate Department at the School of Dentistry of San Martín de Porres University (FO-USMP), Lima, Peru.

2 Dental Surgeon, Specialist in Oral and Maxillofacial Medicine and Pathology, Researcher and Lecturer at the School of Dentistry of San Martín de Porres University (FO-USMP), Lima, Peru.

3 Dental Surgeon, Specialist in Oral and Maxillofacial Radiology, $\mathrm{PhD}$ in Education, Researcher and Lecturer at the School of Dentistry at Universidad of San Martín de Porres University (FO-USMP), Lima, Peru.

${ }^{4}$ Dental Surgeon, PhD in Odontology, Researcher and Lecturer at the School of Dentistry of San Martín de Porres University (FO-USMP), Lima, Peru.

5 Licentiate Degree in Medicine and Surgery, Specialist in Stomatology, $\mathrm{PhD}$ in Odontology, Full Professor of Special Patients at the School of Dentistry at Catalunya International University (UIC), Head of Public Health and Oral Medicine Department, Director of Master's Degree in Gerodontology, Special Patients and with medical commitment, Spain.

${ }^{6}$ Licentiate Degree in Psychology, PhD in Human Development and Family Life. Lecturer at Pontificia Católica del Perú University and University of Kansas.
Received : 04.08.2020

Accepted : 16.10 .2020

How to Cite: Cava-Arangoitia OCJ, Guevara-Canales JO, Morales-Vadillo R, Cava-Vergiú CE, Uribe MR, Ortega LM. Predictive Indicators for Managing Dental Care in Patients with Different Abilities. Cumhuriyet Dent J 2020;23:4: 283-290.

* Corresponding Author:

Calle Pasaje Los Aromos 280 Departamento 301, Urbanización El Remanzo, La Molina, Lima-12, Lima, Peru

E-mail: oswaldocavaa@yahoo.es 


\section{INTRODUCTION}

Patients with different abilities include people who have physical, developmental, mental, sensorial, behavioral, cognitive or emotional impediments. These limiting conditions may require differential medical management, intervention of special medical care and/or use of specialized services or programs. ${ }^{1,2}$ People with different abilities may present high prevalence of oral diseases such as dental caries ${ }^{3}$; possibly due to barriers in communication regarding their own health education and poor oral hygiene habits., The term "different abilities" was coined to focus attention on what these people can do rather than on what they cannot do within the community. ${ }^{6} \mathrm{~A}$ visit to the dentist's office will always be an unwanted experience, which may be frightening or traumatic for many patients ${ }^{7-9}$ The more so for patients with different abilities, who may display a range of reactions, including nervousness, sweating hands, anxiety and even panic.

The different attitudes and behaviors will be more marked at the beginning of dental treatment. This may overwhelm the operator, creating difficulty in making a prognosis for that treatment, which may lead to the operator giving up on providing the patient with an opportunity to be treated while conscious. ${ }^{10}$ It would therefore be ideal and timely to find a way to classify patients according to their behavior in order to predict the possibility of providing oral health care while they are conscious. This is why it is presented detailed dental treatment while conscious for patients with different abilities, based on the application of a new protocol for dividing patients, according to behavior, into Classification (A, B or $\mathrm{C}$ ) and Horizon (I, II, II, IV or V). This classification protocol is based on more than 32 years' experience of the main author of this study in dental care and follow-up of these patients, analysis of the performance of the different personalities, characters and reactions, and the advice and accounts provided by parents about their children. The screening protocol seeks to achieve a planned methodology for providing dental care while the patient conscious, by designing a technique that is simple to apply and which considers the individual characteristics of any patient with different abilities (child, teenager or adult). This would enable the creation of a "Classification and Horizon Screening Protocol according to patient behavior".

The Classification is a division according to behavior for rapid recognition of patient type, by making a good clinical history and considering the family living with the patient. It divides patients into: Group A: Patients who could initially be considered the easiest to treat, with clinical operator working calmly and comfortably without need to resort to physical restraint, conscious sedation or full sedation. Patient is cooperative, extroverted, with self-help skills and with prior positive dental experience. Group B: Patients opposite to Group A, who apparently display very uncooperative behavior and from whose clinical history may be deduced multiple understandable causes for their nervous, restless, bored, intolerant and sometimes aggressive or self-aggressive behavior. Group C: Patients who may belong to Group A or B, but who, due to other reasons, require care in the operating room, e.g., due to serious diseases such as cancer, or to family reasons such as unexpected traveling which requires the treatment to be completed sooner than planned.

The Horizon is a subclassification which does not relate to patient behavior, but to the level of complexity the treatment may reach and whether it is possible to complete it while the patient is conscious. The criterion is to begin with the simplest part and move forward according to complexity, in accordance with patient performance and cooperation. Horizon $\mathrm{I}$ is the simplest level, and Horizon $\mathrm{V}$ is the most complex. Horizon provides information on how far the treatment can advance while the patient it is conscious, to provide quality of life to the patient and family.

This study contributes to the professional theoretical knowledge of dentists by showing what events can influence the management of dental care by means of the new Classification and Horizon screening protocol for patients with different abilities. It makes a social contribution by providing the possibility of these patients not 
being exposed to sedation or general anesthesia, which might involve risks and/or complications. The aim of this study was to determine predictive indicators for managing dental care in patients with different abilities according to a new screening protocol using the indicators classification, horizon, need for readiness prior to dental treatment and type of treatment.

\section{MATERIALS AND METHODS}

\section{Ethical aspects}

The research project was evaluated by the Research Project Reviewing Committee for approval of the Facultad de Odontología, Universidad de San Martín de Porres (FOUSMP), Peru, (Certificate No. 010-2020CRPI/INVE-FO-USMP) and then sent to the Human Research Ethics Committee of FO-USMP (Certificate No. 002-2020-CEI/INVE-FO-USMP), where it secured approval.

The clinical histories of patients who met the inclusion criteria were registered under a code. Researchers undertook to preserve the confidentiality of all data recorded and/or obtained from patients, clinical histories or outcomes, to ensure that they would only be handled by the principal investigator, while the other participating investigators would not have access to them. The investigators declare no conflict of interest.

\section{Methodological design}

This was an observational, analytic, retrospective, cross-sectional study. The sample consisted of patients with different abilities diagnosed with autism, Down syndrome, cognitive deficit, cerebral palsy or other conditions, who received care between 1999 and 2019 at the Dental and Oral Health Service of Centro Ann Sullivan of Peru (CASP) and who met the inclusion criteria. Sample size was approximately 627 patients of whom 589 met the inclusion criteria. Inclusion criteria were having been duly medically diagnosed as having some different ability condition, and receiving dental care at CASP accompanied by their parents on the visit. Exclusion criteria were patients with different abilities whose parents or guardians would not cooperate with the order of the protocol for care of their children and patients with any kind of systemic disease requiring immediate hospitalization.

\section{Data collection}

Data from the clinical histories of patients who met the selection criteria were evaluated. These data were used to identify predictive indicators for managing dental care of the patient with different abilities. The clinical histories of patients medically diagnosed as having different abilities were reviewed, thus providing data related to Classification and Horizon, need for previous readiness and treatment type.

\section{Statistics}

The data recorded on index cards for data collection were transferred to Microsoft Office Excel software (Microsoft, Inc., Redmond, Washington, USA) to create a database.

Categorical variables were described in tables of absolute and relative frequencies, represented in graphs expressed in percentages. The numerical variable Age was described with measures of central tendency and dispersion, and graphed using a histogram. To determine predictive indicators for managing dental care in patients with different abilities, a multivariate statistical analysis was used by means of a binary multiple regression test. Values were considered statistically significant for $\mathrm{p}<0.05$.

Analysis was performed on a computer with Windows ${ }^{\circledR}$ Operative System (Microsoft, Inc., Seattle, Washington, USA) using the SPSS software package version 25 in Spanish.

\section{RESULTS}

627 cases were found to have received care at the CASP Dental and Oral Healthcare Service between 1999 and 2019, of which 38 did not meet the total data required for statistical analysis. Of the 589 cases evaluated, ages ranged from 1.3 to 63 years, mean age was 14.5 years; $67.9 \%$ were male, and the most common systemic diagnosis of different abilities was autism in $62 \%$ of the cases. The most frequent treatment was caries $(52.1 \%)$. Final management of these patients was conscious in $96.4 \%$ of the 589 cases evaluated. Table 1 provides the descriptive data. 
Table 1: Descriptive data of factors that influence the type of dental care management for patients with different abilities

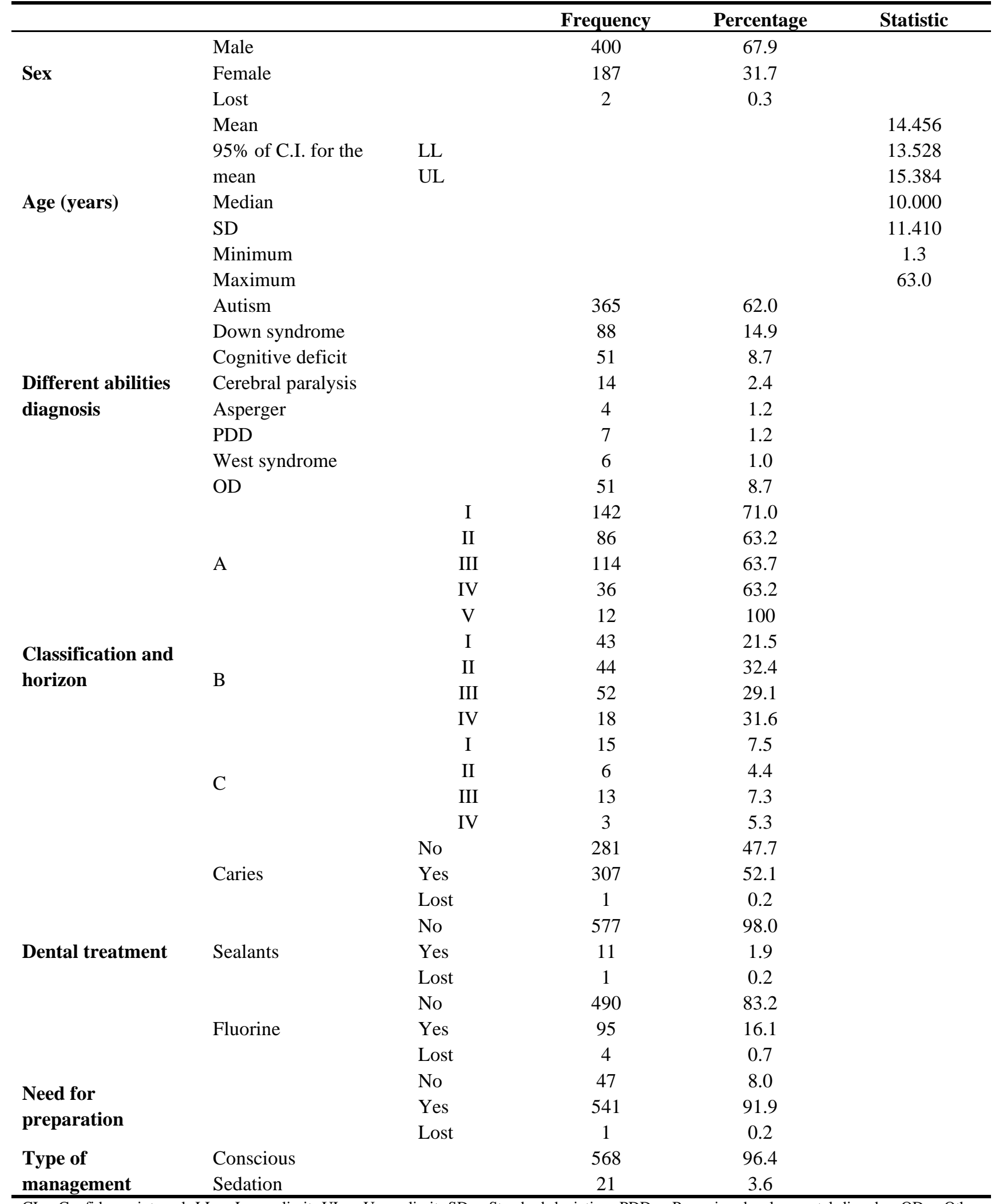

$\mathrm{CI}=$ Confidence interval, $\mathrm{LL}=$ Lower limit, $\mathrm{UL}=$ Upper limit, $\mathrm{SD}=$ Standard deviation, $\mathrm{PDD}=$ Pervasive developmental disorder, OD $=$ Other diagnoses.

Table 2 shows type of Classification and Horizon according to systemic diagnosis, where $67.1 \%$ of the cases corresponded to classification $\mathrm{A}$, with $35.9 \%$ of these in horizon $\mathrm{I}$, and Table 3 show how dental care was managed. 
Table 2: Type of Classification and Horizon according to diagnosis of different abilities

\begin{tabular}{|c|c|c|c|c|c|c|c|c|c|c|c|c|c|c|c|c|c|}
\hline \multirow{2}{*}{\multicolumn{2}{|c|}{$\begin{array}{l}\text { Classification } \\
\text { and Horizon }\end{array}$}} & \multicolumn{2}{|c|}{ Autism } & \multicolumn{2}{|c|}{$\begin{array}{c}\text { Down } \\
\text { syndrome }\end{array}$} & \multicolumn{2}{|c|}{$\begin{array}{c}\text { Cognitive } \\
\text { deficit }\end{array}$} & \multicolumn{2}{|c|}{$\begin{array}{c}\text { Cerebral } \\
\text { palsy }\end{array}$} & \multicolumn{2}{|c|}{ Asperger } & \multicolumn{2}{|c|}{ PDD } & \multicolumn{2}{|c|}{$\begin{array}{c}\text { West } \\
\text { syndrome }\end{array}$} & \multicolumn{2}{|c|}{ OD } \\
\hline & & $\mathbf{N}$ & $\%$ & $\mathbf{N}$ & $\%$ & $\mathbf{N}$ & $\%$ & $\mathbf{N}$ & $\%$ & $\mathbf{N}$ & $\%$ & $\mathbf{N}$ & $\%$ & $\mathbf{N}$ & $\%$ & $\mathbf{N}$ & $\%$ \\
\hline \multirow{5}{*}{$\mathbf{A}$} & I & 102 & 40.5 & 19 & 31.7 & 9 & 34.6 & 2 & 22.2 & 1 & 14.3 & 2 & 40.0 & 1 & 33.3 & 6 & 21.4 \\
\hline & II & 56 & 22.2 & 11 & 18.3 & 5 & 19.2 & 1 & 11.1 & 3 & 42.9 & 2 & 40.0 & 1 & 33.3 & 7 & 25.0 \\
\hline & III & 72 & 28.6 & 16 & 26.7 & 7 & 26.9 & 5 & 55.6 & 2 & 28.6 & 1 & 20.0 & 1 & 33.3 & 10 & 35.7 \\
\hline & IV & 18 & 7.1 & 7 & 11.7 & 5 & 19.2 & 1 & 11.1 & 1 & 14.3 & 0 & 0 & 0 & 0 & 4 & 14.3 \\
\hline & $\mathbf{V}$ & 4 & 1.6 & 7 & 11.7 & 0 & 0 & 0 & 0 & 0 & 0 & 0 & 0 & 0 & 0 & 1 & 3.6 \\
\hline \multirow{5}{*}{ B } & I & 21 & 22.6 & 8 & 36.4 & 3 & 17.6 & 1 & 25.0 & 0 & 0 & 1 & 100 & 1 & 33.3 & 8 & 47.1 \\
\hline & II & 31 & 33.3 & 5 & 22.7 & 3 & 17.6 & 2 & 50.0 & 0 & 0 & 0 & 0 & 1 & 33.3 & 2 & 11.8 \\
\hline & III & 32 & 34.4 & 8 & 36.4 & 5 & 29.4 & 1 & 25.0 & 0 & 0 & 0 & 0 & 1 & 33.3 & 5 & 29.4 \\
\hline & IV & 9 & 9.7 & 1 & 4.5 & 6 & 35.3 & 0 & 0 & 0 & 0 & 0 & 0 & 0 & 0 & 2 & 11.8 \\
\hline & $\mathbf{V}$ & 0 & 0 & 0 & 0 & 0 & 0 & 0 & 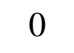 & 0 & 0 & 0 & 0 & 0 & 0 & 0 & 0 \\
\hline \multirow{5}{*}{ C } & $\mathbf{I}$ & 6 & 33.3 & 1 & 33.3 & 5 & 62.5 & 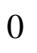 & 0 & 0 & 0 & 1 & 100 & 0 & 0 & 2 & 33.3 \\
\hline & II & 3 & 16.7 & 1 & 33.3 & 0 & 0 & 1 & 100 & 0 & 0 & 0 & 0 & 0 & 0 & 1 & 16.7 \\
\hline & III & 9 & 50.0 & 1 & 33.3 & 1 & 12.5 & 0 & 0 & 0 & 0 & 0 & 0 & 0 & 0 & 2 & 33.3 \\
\hline & IV & 0 & 0 & 0 & 0 & 2 & 25.0 & 0 & 0 & 0 & 0 & 0 & 0 & 0 & 0 & 1 & 16.7 \\
\hline & $\mathbf{V}$ & 0 & 0 & 0 & 0 & 0 & 0 & $\sigma$ & 0 & 0 & 0 & 0 & 0 & 0 & 0 & 0 & 0 \\
\hline
\end{tabular}

PDD = Pervasive developmental disorder, OD = Other diagnoses.

Table 3: Type of dental care management according to diagnosis of different abilities

\begin{tabular}{lcccccc}
\hline & \multicolumn{2}{c}{ Conscious } & \multicolumn{2}{c}{ Sedation } & \multicolumn{2}{c}{ Total } \\
\cline { 2 - 6 } & $\mathrm{N}$ & $\%$ & $\mathrm{~N}$ & $\%$ & $\%$ & $\mathrm{~N}$ \\
\hline Autism & 356 & 62.7 & 9 & 42.9 & 365 & 62.0 \\
Down syndrome & 87 & 15.3 & 1 & 4.8 & 88 & 14.9 \\
Cognitive deficit & 45 & 7.9 & 6 & 28.6 & 51 & 8.7 \\
Cerebral palsy & 14 & 2.4 & 0 & 0 & 14 & 2.4 \\
Asperger & 6 & 1.1 & 1 & 4.8 & 7 & 1.2 \\
PDD & 7 & 1.2 & 0 & 0 & 7 & 1.2 \\
West syndrome & 6 & 1.1 & 0 & 0 & 6 & 1.0 \\
OD & 47 & 8.3 & 4 & 19.0 & 51 & 8.7 \\
\hline PDD = Pervaive
\end{tabular}

PDD $=$ Pervasive developmental disorder, OD = Other diagnoses.

The Forward Wald binary logistic regression method was used for statistical analysis to determine the best statistic predictive model for dental care management. Type of dental health care management was considered as dependent variable, while independent variables were sex, diagnosis, classification, horizon, caries treatment, use of sealants and fluorine application. A 5\% significance level was considered $(p<0.05)$. A significance value of $\mathrm{p}<0.001$ was obtained, which indicates that the model as a whole has significant fit in the second step of introduction of influencing variables using the Forward Wald method. The model reports that patients who require dental care management under sedation are those who have the independent variables "Classification" and "Horizon" as triggering factors. These variables were also found to be 
significantly associated to the dependent variable through a predictive equation with $97.1 \%$ correct classification, described as the probability of using sedation, as follows:

$$
\mathrm{Y}=-17.151+13.898(\text { Horizon I) }+14.301
$$

(Horizon II) +16.487 (Horizon III) +17.469

(Horizon IV) + 1 (Horizon V) - 4.051 (Classification A) - 1.431 (Classification B) +1 (Classification C).

Probability of using sedation $=\frac{1}{1+e^{-y}}$

In addition, regression analysis showed that our model has high sensitivity $(100 \%)$ and low specificity (15\%), so it adequately classifies patients who do not require sedation and deficiently classifies patients who do require sedation.

\section{DISCUSSION}

In 2018, the World Health Organization estimated that over a billion people (about $15 \%$ of the world population) have some form of disability. ${ }^{11}$ This is higher than the previous WHO estimate, which was $10 \%$ in the 1970s. Thus, disability is part of the human condition, since nearly everyone, at some time in life, will have a temporary or permanent disability. ${ }^{6}$

With regard to oral health, Faulks et al. ${ }^{12}$ have defined people who require special dental care as those with a disability or restriction to activity which directly or indirectly affects their oral health, within their personal and environmental context, using the International Classification of Functioning, Disability and Health model.

Many studies from different parts of the world have established that people who require special dental care have poor oral health and high levels of unmet needs in terms of prevention and periodontal, restorative and functional treatment. ${ }^{13-16}$ Indeed, dental care is one of the most frequent unmet health needs for people with special needs. ${ }^{17,18}$

For many people with intellectual or developmental disabilities, available treatment consists mainly of emergency extractions, often under physical immobilization ${ }^{19}$ and general anesthesia, rather than comprehensive planned care. ${ }^{14,20,21}$

Patients included among those with special needs should have access to a dental home for primary oral health care. This refers to the continuous relationship between that patient and his/her dentist, including all aspects of oral health, provided in a comprehensive, accessible, coordinated, family-centered manner. ${ }^{22}$

Dentists may lack some knowledge and skills required to meet the oral health needs of these patients, which may explain their lack of confidence and unfavorable attitudes, and may be related to the time spent with these patients or to being limited to merely symptomatic, rather than comprehensive treatment. ${ }^{23}$ Thus, dentists who regularly manage patients with special needs could be a source of relevant information for research into obstacles and recommendations on how to overcome those obstacles. ${ }^{22}$ In addition, the specialty Dentistry for Special Patients should be recognized, to enable seamless primary and secondary oral healthcare services to be organized and provided to these people. ${ }^{24}$ Unfortunately, most undergraduate programs in North America do not include minimum didactic and practical clinical experience in the care of people with disabilities..$^{25}$

When a patient is treated while conscious, he/she is not exposed to any kind of sedation which could involve some level of risk. The key to success is to provide friendly care by learning about patient's motivations, e.g., finding out about a patient's favorite music and playing it during the clinical session, or giving the patient a warm welcome. Thus, from 1982 to 2014 in Peru, dental treatment for people with different abilities performed in operating rooms and under general anesthesia has descended from $95 \%$ to $4 \% .^{26}$

\section{CONCLUSIONS}

The results show that in almost all the cases considered, dental care was managed while the patient with different abilities was conscious, and this is significantly influenced by the indicator provided by the division regarding behavior and the level of complexity in the dental treatment, 
called the Classification and Horizon Screening Protocol. This indicator provided the best prediction for whether or not sedation was needed, and the model adequately classifies patients who do not require sedation.

\section{ACKNOWLEDGEMENT}

None

\section{CONFLICT OF INTERESTS}

The authors declare no conflict of interest with potential applications or effects of the results of this research.

\section{REFERENCES}

1. Largent BA. The American Academy of Pediatric Dentistry and the specialty of pediatric dentistry. J Am Coll Dent 2009;76:18-22.

2. van Dyck PC, Kogan MD, McPherson MG, Weissman GR, Newacheck PW. Prevalence and characteristics of children with special health care needs. Arch Pediatr Adolesc Med 2004;158:884-890.

3. Frank M, Keels MA, Quiñonez R, Roberts M, Divaris K. Dental Caries Risk Varies Among Subgroups of Children with Special Health Care Needs. Pediatr Dent 2019;41:378-384.

4. Jain M, Mathur A, Kumar S, Dagli RJ, Duraiswamy

P, Kulkarni S. Dentition status and treatment needs among children with impaired hearing attending a special school for the deaf and mute in Udaipur, India. J Oral Sci 2008;50:161-165.

5. Buldur B. Pathways between parental and individual determinants of dental caries and dental visit behaviours among children: Validation of a new conceptual model. Community Dent Oral Epidemiol 2020;48:280-287.

6. Mayo-Ortega L, LeBlanc JM. Inclusion across the life span for people with different abilities. In: Timmins V, Walsh P, editors. A Long Walk to School, Global Perspectives on Inclusive Education. Rotterdam, Boston, Taipei: Sense Publishers; 2003. p. 27-39.

7. Buldur B, Armfield JM. Development of the Turkish version of the Index of Dental Anxiety and Fear (IDAF-4C+): Dental anxiety and concomitant factors in pediatric dental patients. J Clin Pediatr Dent 2018;42:279-286.
8. Rantavuori K, Zerman N, Ferro R, Lahti S. Relationship between children's first dental visit and their dental anxiety in the Veneto Region of Italy. Acta Odontol Scand 2002;60:297-300.

9. Buldur B. Behavior Management in Pediatric Dentistry: An Overview and Interpretation. Pesqui Bras Odontopediatria Clín Integr 2019;19:e4649.

10. Buldur B, Armfield JM. Perceptions of the dental profession: a comparative analysis through scale development. Eur J Oral Sci 2018;126:46-52.

11. World Health Organization. World report on disabilities. [Internet] 2018 [cited 21 Jun 2020]. Available at: https://www.who.int/news-room/factsheets/detail/disability-and-health.

12. Faulks D, Hennequin M. Defining the population requiring special care dentistry using the International Classification of Functioning, Disability and Health - a personal view. J Disabil Oral Health 2006;7:143-152.

13. Anders PL, Davis EL. Oral health of patients with intellectual disabilities: a systematic review. Spec Care Dentist 2010;30:110-117.

14. Matevosyan NR. Oral health of adults with serious mental illnesses: a review. Community Ment Health J 2010;46:553-562.

15. Faulks D, Freedman L, Thompson S, Sagheri D, Dougall A. The value of education in special care dentistry as a means of reducing inequalities in oral health. Eur J Dent Educ 2012;16:195-201.

16. Morgan JP, Minihan PM, Stark PC, Finkelman MD, Yantsides KE, Park A, et al. The oral health status of 4,732 adults with intellectual and developmental disabilities. J Am Dent Assoc 2012;143:838-846.

17. Crall JJ. Improving oral health for individuals with special health care needs. Pediatr Dent 2007;29:98104.

18. Sigal A, Sigal MJ. Overview of a hospital based dental programme for persons with special needs. J Disabil Oral Health 2006;7:176-184.

19. Salles PS, Tannure PN, Oliveira CA, Souza IP, Portela MB, Castro GF. Dental needs and management of children with special health care needs according to type of disability. J Dent Child (Chic) 2012;79:165169. 
20. Gabre P, Martinsson T, Gahnberg L. Incidence of, and reasons for, tooth mortality among mentally retarded adults during a 10-year period. Acta Odontol Scand 1999;57:55-61.

21. Pradhan A, Slade GD, Spencer AJ. Factors influencing caries experience among adults with physical and intellectual disabilities. Community Dent Oral Epidemiol 2009;37:143-154.

22. Medical Home Initiatives for Children With Special Needs Project Advisory Committee. American Academy of Pediatrics. The medical home. Pediatrics 2002;110:184-186.

23. Blaizot A, Catteau C, Delfosse C, Hamel O, Trentesaux T. Obstacles to comprehensive dental care in patients with sustained limitations of their decisionmaking abilities: findings from a Delphi study. Eur J Oral Sci 2018;126:222-233.
24. Lewis D, Fiske J, Dougall A. Access to special care dentistry, part 7. Special care dentistry services: seamless care for people in their middle years--part 1 . Br Dent J 2008;205:305-317.

25. Wolff AJ, Waldman HB, Milano M, Perlman SP. Dental students' experiences with and attitudes toward people with mental retardation. J Am Dent Assoc 2004;135:353-357.

26. Mayo-Ortega L, LeBlanc JM. South America. A service example from Lima, Peru. In: Rubin IL, Merrick J, Greydanus DE, Patel DR, editors. Health Care for people with intellectual and developmental disabilities across the lifespan. 3rd ed. Dordrecht, Netherlands: Springer International Publishing Switzerland; 2016. p. 517-528. 